\title{
Chapter 18 \\ A Practical Approach to Digital \\ Transformation: A Guide to Health \\ Institutions in Developing Countries
}

\author{
Alvin B. Marcelo
}

\begin{abstract}
Most healthcare organizations, at the local, national, and international levels aspire to commence their digital transformation but are at a loss on how to start the process. This chapter presents a practical approach that begins with laying down the foundations for strong governance to guide institutions towards this complex process. The approach begins with Governance $(\mathrm{G})$ - setting clear decision-making structures and strategic directives to the whole enterprise. This is followed by adoption of Frameworks $(\mathrm{F})$ that provide a common reference for all stakeholders as they undergo their respective changes. Because almost all healthcare data are sensitive and should be kept confidential, Ethical (E) processes must be in place to ensure that patients are safe and that their welfare is of the utmost priority. Data governance (D) then comes into play providing clear guidelines, systems, and structures in the management of data. Once these aforementioned fundamentals are in place, cloud and compliance $(\mathrm{C})$ capabilities should be available to ensure that a secure infrastructure is in place to store, process, and protect large volumes of information. This elastic infrastructure enables the accumulation of big data (B) at a rate faster than what most analytical tools can manage in real-time opening up opportunities for visualizing information. With this tremendous amounts of data, the prerequisits are laid out for Artificial Intelligence (A) and new insights, previously unknown, can be discovered and used for creating new products and services for the enterprise and as input for decision-making for improved governance.
\end{abstract}

Keywords Digital health $\cdot$ Governance $\cdot$ Compliance $\cdot$ Ethics

\section{Learning objectives}

By the end of this chapter, you will be able to

- understand a practical framework for introducing technology-based changes in health institutions in low-to-medium income countries (LMICs)

- present an incremental approach to implementing business transformation through a sequence of interventions designed to gradually introduce these changes

\footnotetext{
A. B. Marcelo $(\square)$

University of the Philippines Manila, Manila, Philippines

e-mail: admarcelo@up.edu.ph
} 
- deliver benefits at every step of the approach to ensure a strong foundation for the next one.

\subsection{Context}

On September 2015, world leaders adopted the seventeen Sustainable Development Goals (SDGs) of the 2030 Agenda for Sustainable Development. ${ }^{1}$ From January 1, 2016, the new Goals will replace the Millennium Development Goals and aim to go further and end all forms of poverty. Quality of life is expected to improve and more people will have longer lives. Along with these longer lifespans however, come chronic diseases which can overwhelm human resources for health. Mollura (2014) ${ }^{2}$ predicts that this mismatch of patients and providers will require new technologies such as artificial intelligence to respond to the increased demand for healthcare services.

Prior to this in 2011, the World Health Organization created the Asia eHealth Information Network (AeHIN) to support national efforts at developing eHealth at national scale. The network started from a small group of representatives from Ministries of Health who understood that national health information systems will benefit from digitization but realized they lacked knowledge and skills to achieve it. As the group enlarged in number, its members shared knwoledge and crafted a position paper on how to move forward and face these challenges. (Marcelo and Kijsanayotin).

At their 2012 general meeting, AeHIN introduced the National eHealth Strategy Toolkit, ${ }^{3}$ jointly released by the World Health Organization and the International Telecommunications Union. It was a guide for developing countries desiring to build comprehensive health information systems. Figure 18.1 shows the seven core components of the Toolkit.

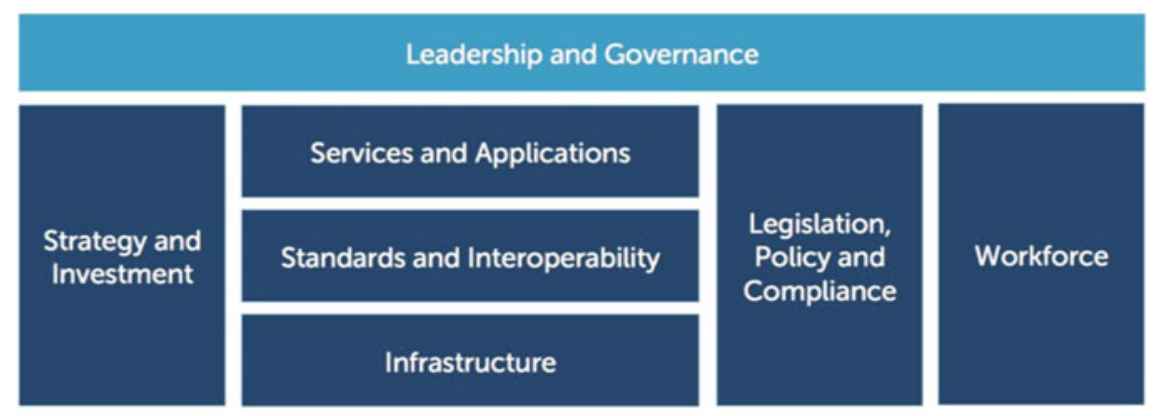

Fig. 18.1 The seven core components of the WHO-ITU national eHealth strategy toolkit

\footnotetext{
${ }^{1}$ United Nations Department of Economic and Social Affairs (2016).

${ }^{2}$ Mollura et al. (2017).

${ }^{3}$ National eHealth Strategy Toolkit [WWW Document] (n.d.).
} 
While the WHO-ITU Toolkit helped identify the core components of a national eHealth strategy, it did not prescribe a process for national eHealth development beyond emphasizing the primacy of leadership and governance.

Faced with this limitation, AeHIN network leaders embarked on a capacitybuilding program on an ad hoc basis-first taking courses on Health Level 7 (HL7) - a standard for exchanging health data.

Finding HL7 insufficient for large scale interoperability, additional training was obtained on enterprise architecture using The Open Group Architecture Framework or TOGAF. TOGAF is a standardized process for developing blueprints for information systems that can guide developers how to use HL7 and other standards properly for effective data exchange. Finally, realizing that an enterprise architecture or blueprint is only effective if complied with by all stakeholders, the network members then sought training on IT governance using COBIT5. COBIT5 is a business framework for the governance and management of enterprise information technology. COBIT5 ensures that stakeholders in an enterprise are properly identified and their benefits, risks, and resources are taken into consideration when developing information systems that will affect them. In retrospect, after sending several cohorts to the aforementioned trainings, the Network realized that a better and more systematic sequence was the reverse: IT governance should come first, followed by enterprise architecture, then program management and standards. This resulted in AeHIN's Mind the GAPS slogan.

Concurrently, the Digital Impact Alliance (DIAL) published the SDG Digital Investment Framework ${ }^{4}$ which promotes the adoption of a whole-of-government approach in technology investment together with a theory of change, refer to in Fig. 18.2. This framework followed a process where a theory of change served as basis for a theory of action.

\section{SDG Digital Investment Framework Theory of Change}

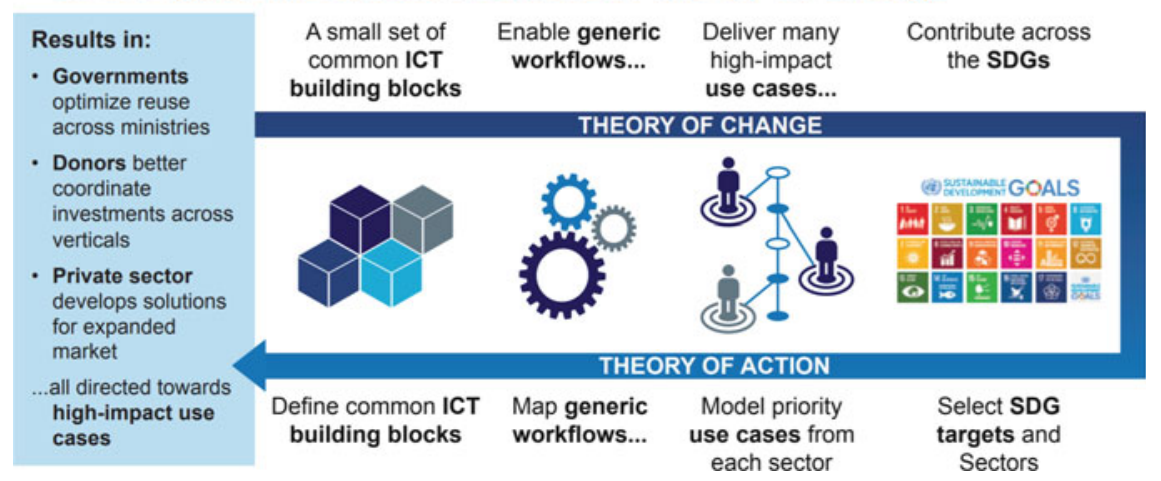

Fig. 18.2 Digital impact alliance theory of change for the sustainable development goals

\footnotetext{
${ }^{4}$ SDG Digital Investment Framework-A whole-of-Government Approach to Investing in Digital Technologies to Achieve the SDGs [WWW Document] (n.d.).
} 


\subsubsection{Artificial Intelligence and health systems}

Recently, the topic of artificial intelligence (AI) has gained popularity in the AeHIN mailing list, and several members have indicated an interest in understanding how they can receive its benefits. With the decreasing cost of hardware and easy availability of cloud-based applications, the accumulation of data has progressed substantially. There was increasing interest from the network members to understand if the body of data could provide insight into patterns of healthcare that could be leveraged to improve service delivery and eventually, better patient outcomes.

Looking at the current GAPS concepts, it was evident that the framework can be used for both national level interventions and institutional level implementation. This alignment is very important because data accrued at the institutional level and gains power when combined at a larger scale. This opened up the opportunity to develop a more practical approach that can guide institutions in the process of digital transformation that contributes to national development.

This approach combines the AeHIN and DIAL methodologies at the institutional level and offers a simple stepwise methodology for strategic IT investments that can lead to the adoption of artificial intelligence for achieving better health.

With DIAL's cyclical theory of change and theory of action, stakeholders are linked through their common desire to achieve the Sustainable Development Goals and the use of ICT building blocks. These shared objectives provide the alignment of stakeholders and enable the collaboration required to succeed with their respective SDG agenda. AeHIN's Mind the GAPS, on the other hand, adopts industry methodologies to achieve the same (COBIT5, TOGAF, PRINCE2, HL7).

\subsection{Governance}

The approach, like AeHIN's, begins with governance which ensures that there is a clear and accepted strategy and decision-making framework respected by all stakeholders. The strategy is crucial to set common directions for the enterprise guided by a vision established by the most accountable entity in the organization.

In most for-profit enterprises, this accountable entity is the board of directors. In public office, the governance will be formed from elected officials. Whatever the constitution, the eventual governance structure must be respected by all stakeholders. Figure 18.3 shows the roles, activities, and relationships of various stakeholders according to COBIT5. ${ }^{5}$ In the Philippines for example, the Department of Health led the formation of a multi-sectoral National eHealth Steering Committee

${ }^{5}$ COBIT 5: A Business Framework for the Governance and Management of Enterprise IT [WWW Document] (n.d.). 


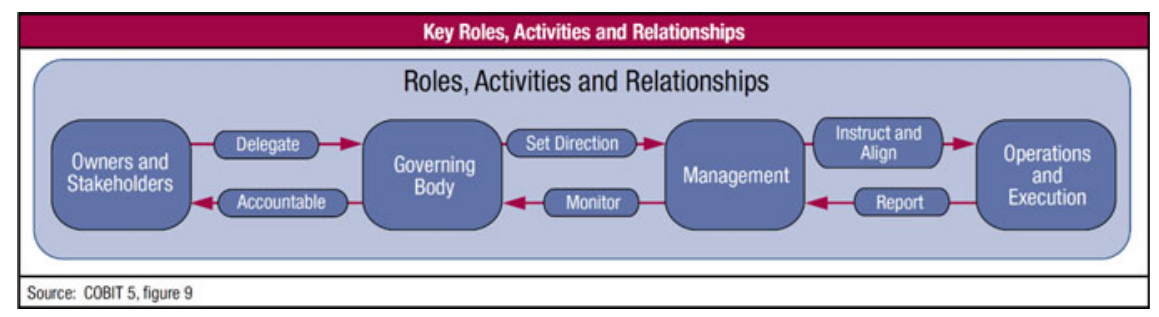

Fig. 18.3 Sequence of roles, activities and relationships according to COBIT5

composed of Ministers of ICT, Ministers of Science and Technology, the president of the National Health Insurance Corporation and the National Health Sciences University to provide directions to the national eHealth program. ${ }^{6}$

Once formed, the main responsibility of the governing body is to set directions for the organization-to ensure that benefits are defined clearly for the whole organization, risks are quantified and monitored, and resources are adequate and available.

After that, another priority responsibility of the governing body is to adopt a framework. Framework setting and maintenance are important high-level activities especially in complex environments where numerous components and stakeholders are involved. For information systems, several IT governance frameworks are available such as ISO $38500^{7}$ (Corporate Governance of Information Technology), COBIT5, and ISO TR $14649^{8}$ (Capacity-based eHealth architecture roadmap). While these frameworks differ by degrees of detail, they eventually map to each other. ISO TR 14639 focuses specifically on national eHealth architectures while ISO 38500 is generalizable to any enterprise. COBIT5 straddles between the two and contains more details than ISO 38500 but with more generic processes compared to ISO TR 14639 , refer to figure.

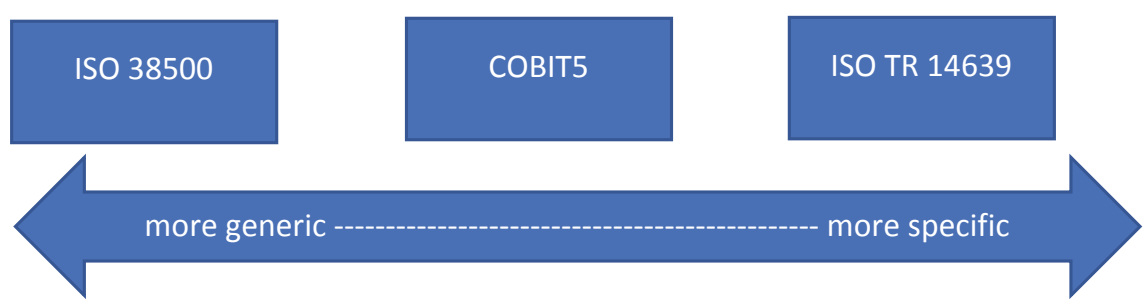

\footnotetext{
${ }^{6}$ Department of Health, Republic of the Philippines. Reconsitution of the National eHealth Steering Committee.

${ }^{7}$ 14:00-17:00 (n.d.).

${ }^{8}$ 14:00-17:00 (n.d.).
} 


\subsubsection{Ethics in Health IT Governance}

One important risk with information systems, especially those that manage personal health information, are breaches. Because health data are sensitive, ethical protocols must be enforced to ensure the privacy of subjects. There are data protection protocols available from the National Institutes of Health, and digital transformation advocates in the health sector are advised to submit themselves to the institutional ethics review process in order to protect health data and maintain the trust of their patients. Courses such as CITI Program's "Data and Specimen Only"9 research training specify the requirements for securing sensitive data and the responsibility of ethics review boards to provide the assurance that investigators follow these guidelines.

In anticipation of the rapid accumulation of data, institutions must invest on data governance and data management. Data governance is important to ensure consistent understanding within the organizations on the value of data and its contribution to corporate strategy. Data management, on the other hand, encompasses the policies, procedures and technologies required to achieve this. The Data Management Association (DAMA) publishes its Body of Knowledge (DAMA-BOK) ${ }^{10}$ which can be used by institutions to harness the power of their data to strategic advantage.

While the data management systems are being set up, concurrent efforts to prepare the necessary policies and infrastructure to store large volumes of data must be made. ISO/IEC $17788^{11}$ lists features of cloud technology that enable organizations to gather structured and unstructured data and securely store them in easily expandable form. In addition, cloud technology is able to rapidly provision computing resources as needed providing elasticity to users. This responsiveness removes the delays in procurement traditionally attributed to the the IT department. Whereas previously procurement was a slow process that hindered access to computational power, cloud technology eliminates that by allowing authorized users to request resources on demand.

This elasticity is a crucial pre-requisite for the collection of large amounts of data or big data. When large digital assets are in the cloud, this opens up the need for visualization techniques that enable users to understand data that is accumulating at a rapid rate (velocity), in diverse formats (variety), and in size (volume). These visualization techniques help researchers understand the patterns from the corpus and enable them to obtain new insights from the data. Specifically, visualizations help non-technical health professionals understand the key message from a large dataset that would've been difficult to understand. Presenting large volumes of data as images also helps analysts focus on the message and makes obvious certain parameters (example: changing patterns over time) that are otherwise difficult to see through plain text or tables. ${ }^{12}$

\footnotetext{
${ }^{9}$ Final Rule Material (2017).

${ }^{10}$ Body of Knowledge (n.d.).

${ }^{11}$ 14:00-17:00 (n.d.).

${ }^{12}$ AHIMA Staff. (n.d.).
} 
With the large amounts of managed data, institutions now have the requisites to embark on artificial intelligence. First described by John McCarthy in $1956^{13}$ as "a study...to proceed on the basis of conjecture that every aspect of learning or any other feature of intelligence can in principles be so precisely described that a machine can be made to simulate it", artificial intelligence requires a corpus of structured and unstructured data to simulate human perception. Unlike big data which attempts to understand patterns, artificial intelligence goes beyond that and becomes "a machine.. (that) imitate(s) intelligent human behavior." It is this simulation of human behavior aspect of AI that differentiates it from other emerging technologies previously discussed.

\subsection{Conclusion}

In summary, healthcare institutions in developing countries in Asia are challenged with how they can take advantage of the increasing digitization of society. With the proposed practical approach, institutions can easily remember the steps that starts with the most important governance function followed by the sequential laying down of solutions such as frameworks, ethics, data governance, cloud, big data, and artificial intelligence. While artificial intelligence may be beyond the capabilities of most developing countries at the moment, adopting this practical approach can help them incrementally build capacity enabling them to reap benefits while taking risks that they can manage. In the end, even if the journey towards AI may take time, institutions strategically develop their systems in a direction that makes achieving it more possible.

\section{References}

14:00-17:00. (n.d.). ISO/IEC 17788:2014 [WWW Document]. ISO. Retrieved April 222019 from http://www.iso.org/cms/render/live/en/sites/isoorg/contents/data/standard/06/05/60544.html.

14:00-17:00. (n.d.). ISO/IEC 38500:2015 [WWW Document]. ISO. Retrieved April 222019 from http://www.iso.org/cms/render/live/en/sites/isoorg/contents/data/standard/06/28/62816.html.

14:00-17:00. (n.d.). ISO/TR 14639-1:2012 [WWW Document]. ISO. Retrieved April 222019 from http://www.iso.org/cms/render/live/en/sites/isoorg/contents/data/standard/05/49/54902.html.

AHIMA Staff. (n.d.). The rise of healthcare data visualization I Journal of AHIMA. Retrieved July 9, 2019, from https://journal.ahima.org/2017/12/21/the-rise-of-healthcare-data-visualization/.

Body of Knowledge I DAMA [WWW Document]. (n.d.). Retrieved April 222019 from https:// dama.org/content/body-knowledge.

COBIT 5: A Business Framework for the Governance and Management of Enterprise IT [WWW Document]. (n.d). Retrieved April 22, 2019 from http://www.isaca.org/COBIT/Pages/COBIT-5. aspx.

\footnotetext{
${ }^{13}$ Rajaraman (2014).
} 
Department of Health, Republic of the Philippines. Reconstitution of the National eHealth Steering Committee. Retrieved from http://ehealth.doh.gov.ph/index.php/82-reconstitution-ofthe-national-ehealth-steering-committee.

Final Rule Material. (2017). Secondary Research with Identifiable Information and Biospecimens. Riddle.

Mollura, D. J., Soroosh, G., \& Culp, M. P. (2017). RAD-AID conference writing group, 2017. 2016 RAD-AID conference on international radiology for developing countries: Gaps, growth, and United Nations sustainable development goals. Journal of the American College of Radiology, 14, 841-847. Retrieved from https://doi.org/10.1016/j.jacr.2017.01.049.

National eHealth Strategy Toolkit [WWW Document]. (n.d.). Retrieved April 22, 2019, from https:// www.itu.int/pub/D-STR-E_HEALTH.05-2012.

Rajaraman, V. (2014). JohnMcCarthy-Father of artificial intelligence. Resonance, 19, 198-207. https://doi.org/10.1007/s12045-014-0027-9.

SDG Digital Investment Framework-A whole-of-Government Approach to Investing in Digital Technologies to Achieve the SDGs [WWW Document]. (n.d.). Retrieved April 22, 2019 from https://www.itu.int/pub/D-STR-DIGITAL.02-2019.

United Nations Department of Economic and Social Affairs. (2016). The Sustainable Development Goals Report 2016, The Sustainable Development Goals Report. UN. Retrieved from https://doi. org/10.18356/3405d09f-en.

Open Access This chapter is licensed under the terms of the Creative Commons Attribution 4.0 International License (http://creativecommons.org/licenses/by/4.0/), which permits use, sharing, adaptation, distribution and reproduction in any medium or format, as long as you give appropriate credit to the original author(s) and the source, provide a link to the Creative Commons license and indicate if changes were made.

The images or other third party material in this chapter are included in the chapter's Creative Commons license, unless indicated otherwise in a credit line to the material. If material is not included in the chapter's Creative Commons license and your intended use is not permitted by statutory regulation or exceeds the permitted use, you will need to obtain permission directly from the copyright holder. 